\title{
The Role of Starting Points to Order Investigation: Why and How to Enrich the Logic of Research Questions
}

\author{
William C. Bausman*
}

\begin{abstract}
What methodological approaches do research programs use to investigate the world? Elisabeth Lloyd's Logic of Research Questions (LRQ) characterizes such approaches in terms of the questions that the researchers ask and causal factors they consider. She uses the Logic of Research Questions Framework to criticize adaptationist programs in evolutionary biology for dogmatically assuming selection explanations of the traits of organisms. I argue that Lloyd's general criticism of methodological adaptationism is an artefact of the impoverished LRQ. My Ordered Factors Proposal extends the LRQ to characterize approaches with sequences of questions and factors. I highlight the importance that ordering one's investigation plays in approaches at the level of adaptationism by analyzing two research programs in community ecology: competitionists and neutralists. Competitionists and neutralists take opposed starting points and use explanatory and developmental heuristics to consider more factors in due time. On the Ordered Factors Proposal, these approaches are not only the ecological factors they are open to considering but also the order in which they will consider them. My disagreement with Lloyd's over how to characterize methodological approaches reflects different views about methodological monism and pluralism.
\end{abstract}

Keywords

evolution • ecology • Logic of Research Questions • methodology • research program • adaptationism $\bullet$ neutralism

\section{Introduction}

Scientists decide on what methodology to use to approach their problems. High-level methodologies such as adaptationism in evolution have been denounced by some for hindering progress as they are commended by others for accelerating it. As methodologies serve to organize and

*Department of Philosophy, University of Wisconsin, Madison, USA, wcbausman@gmail.com

(iD) https://orcid.org/0000-0002-7445-8722

Received 30 April 2020; Revised 22 February 2021; Accepted 9 July 2021

doi:10.3998/ptpbio. 2100 
drive scientific research projects and programs, the epistemology of science deserves a framework to characterize and critique them. Such a framework can then itself aid in better understanding why and how methodologies inhibit and enable investigation.

Elisabeth Lloyd (2015, 2016; Lloyd and Oreskes 2018) developed the Logic of Research Questions (LRQ) framework as a tool to characterize and critique the methodological approaches that research programs use across biology and climate science in terms of questions and answers. She has deployed the Logic of Research Questions to show that several adaptationist research programs in behavioral evolutionary biology and evolutionary psychology research are problematic because of the very questions asked and the answers sought. But Lloyd also uses the LRQ to characterize and critique methodological approaches more generally. According to Lloyd, all programs in evolutionary biology that use the methodological adaptationist approach assume out the outset that the traits under investigation have a function. Therefore, all adaptationist programs should be rejected for not being open to considering all the other known evolutionary causes. Lloyd advocates the Evolutionary Factors Framework approach which makes no such assumption.

Lloyd aims for the LRQ to be useful for scholars of science when analyzing research programs and investigative methodologies beyond adaptationism. But to be a useful tool, the LRQ needs to be straightforward to apply to new cases. In this paper I argue for extending the LRQ with the Ordered Factors Proposal by challenging Lloyd's general argument against methodological adaptationism. The LRQ assumes that methodological approaches are characterized by single questions. This entails that there is no way to distinguish objectionable from acceptable forms of adaptationism while also distinguishing among other acceptable approaches. To show this I draw on a case study of two approaches used in community ecology which lack the historical associations of adaptationism. Competitionists and neutralists order their investigation using starting points just like acceptable adaptationists. The Ordered Factors Proposal then characterizes approaches using ordered sequences of factors. In addition to making room with the LRQ for acceptable methodological adaptationism, my proposal avoids the consequence that there is only one acceptable approach to studying evolution.

\section{The Logic of Research Questions}

The LRQ is a tool for characterizing the methodological approaches biologists undertake in terms of questions and answers. The scientific domain for the LRQ is potentially all of science, but Lloyd focuses primarily on biology and especially the form and distribution of organismal traits, the plurality of evolutionary factors affecting these traits, and the research programs investigating the factors affecting the traits.

Lloyd presents the LRQ by contrasting two approaches: the Evolutionary Factors Framework and methodological adaptationism. Some research programs should be characterized as methodological adaptationist (MA) by the LRQ, asking the dogmatic question, What is the function of this trait? Permissible answers to this question are all of the form "The function of the trait is $X$." Therefore, biologists using MA assume natural selection to be responsible and fail to consider the responsibility of other evolutionary factors.

Lloyd proposes an alternative methodology meant to rectify adaptationism's selective blindness. The Evolutionary Factors Framework (EFF) asks the ecumenical question, What evolutionary factors account for the form and distribution of this trait? According to the logic of questions, permissible answers to this question are of the form, The evolutionary factors that account for the form and distribution of this trait are $X, Y, Z$, etc., where $X, Y, Z$ are different evolutionary factors including: natural selection, sexual selection, genetic linkage, phyletic his- 
tory, developmental factors, drift, embryological constraints, social, environmental, and niche coevolutionary factors, etc. (Lloyd 2015, 343). That is, biologists using the EFF are open to considering all known evolutionary factors as potentially responsible.

After distinguishing their questions, Lloyd presents a powerful and simple argument against research programs ever using MA and for always using the EFF.

\section{Evolutionary Factors Argument}

1. All evolutionary research programs using the MA approach are open to considering only a subset of known evolutionary factors.

2. No evolutionary research program should be open to considering only a subset of known evolutionary factors.

3. Therefore, no evolutionary research program should use methodological adaptationism.

4. Instead, all research programs studying evolution should be open to considering all known factors and use the EFF approach.

I deny that premise 1 applies to all approaches which should be considered methodological adaptationist and I resist inferring conclusion 4 from premises 1 and 2. I agree with Lloyd's critiques of the particular research programs guilty of pursuing natural selection as the only potential cause in evolutionary biology. But Lloyd does more than critique particular adaptationist programs, she argues that methodological adaptationism qua methodological approach should be abandoned. This general claim follows if all methodological adaptationists dogmatically assume all traits have functions despite all evidence to the contrary. But I am not convinced that methodological adaptationism is itself the root of the problems and so I am not convinced that the LRQ appropriately characterizes all methodological adaptationist programs as MA. I aim to make space within the logic of research questions for some methodological adaptationists not using MA and for a plurality of good approaches to capture the actual methodological diversity in biology.

To pursue my critique of LRQ I raise the issue of how to classify programs who temporarily or at first pursue natural selection as the cause of investigated traits. I begin by posing a dilemma to the LRQ as it stands now. I then analyze Lloyd's texts to show that one can find support in Lloyd's texts for both horns of the dilemma, making the results of using the LRQ to classify the selection-first approaches indeterminate.

\section{Who Is a Methodological Adaptationist?}

Lloyd developed the LRQ to address the problematic programs across biology which only seek to evidence adaptive hypotheses. Ernst Mayr (1983) defended methodological adaptationism in response to Gould and Lewontin's (1979) famous critique. For Lloyd, Mayr's research approach is MA because he advocates always starting the investigation of the cause of some trait in a population with the hypothesis that it was caused by selection. Mayr says that it is only after selection cannot be shown that chance hypotheses (which seem to stand in for all other hypotheses) have tentative warrant and should be pursued.

The common thread in defenses of methodological adaptationism that Lloyd traces all follow Mayr in advocating looking for selection first and then looking for other hypotheses. Lloyd finds that many biologists who claim to be open to alternative hypotheses after selection hypotheses are not in practice open to considering alternatives. And this, she thinks, is the general feature 
of methodological adaptationism, which is why she uses the LRQ to classify them as MA, asking "What is the function of this trait?" This question is inherently problematic because it fails to consider almost all evolutionary causes in acceptable answers.

The issue I want to address is whether Lloyd is correct in general to classify the approaches of research programs which begin with searching for adaptationism as MA in the LRQ. I agree with Lloyd that, given the LRQ, MA is always bad. But I am not convinced that all methodological adaptationists should be classified as MA.

The advocate for the LRQ faces a dilemma that Lloyd is implicitly aware of insofar as she gives reasons to come down on both sides. The two opposed theses are:

\section{Dilemma 1}

1. All methodological adaptationists use MA.

2. Some methodological adaptationists use EFF.

\section{In Favor of (1) All Methodological Adaptationists use $M A$.}

And philosophers and biologists have argued that there is a certain approach to adaptationism, under which one just assumes temporarily that the trait is an adaptation, and proceeds from there, known as methodological adaptationism, that is both productive and harmless. I think this is false. Adaptationism is not harmless, even in its weakened methodological form. (Lloyd 2016, 130)

Lloyd clearly states the thesis that she objects to: adaptationism which looks for selection first is itself objectionable. The contrast is with an openly dogmatic adaptationism which outright claims to never look beyond selection hypotheses. But for Lloyd (2015), the selection-first adaptationism is itself problematic and objectionable:

it is still an open question whether the method outlined for the methodological adaptationist in practice ever allows non-adaptive explanations ever to win the day. (346)

The reason why she finds the selection-first adaptationists objectionable is that she doubts that they ever actually consider other evolutionary factors. In both this and the previous quotation, she is writing generally about methodological adaptationism and its characterization by the LRQ, not making claims about particular research programs.

Since Mayr and other methodological adaptationists admit the possibility of nonadaptive alternatives, it would seem that they admit, at least in lip service, that there should be nonadaptive answers on the list of possible answers to their question. Should nonadaptive answers belong on the methodological adaptationist list? Should the methodological adaptationist list perhaps look like the evolutionary factors list, but with a few more entries like: 'The function of this trait is $P$ '? The answer to both of these questions is no. (346)

Lloyd here considers the possibility that some methodological adaptationists should be classified as using EFF in LRQ. And she rejects the possibility for reasons that she argues for later in the paper. I will address her strongest reasons below in section 7. In short, she thinks that methodological adaptationism lacks a stopping rule telling them to move on when enough is enough.

\footnotetext{
"MA" and "EFF" will refer to their classification within the LRQ (by Lloyd usually). I make it clear when I am using "methodological adaptationist" in a non-LRQway.
} 
Secondly, if, in attempting to pursue the evolutionary factors methodology, the research community gets stuck on the first, adaptationist step of pursuing only functional answers to the question of whether the trait has a function, then the other characterizations of the trait will not be pursued, and a balanced view of the weight of evidence will not appear. Logically, they would be behaving as methodological adaptationists. (Lloyd 2016, 150)

Here Lloyd makes the point that what matters is not what questions the researchers say that they ask but only the question they actually ask and so the answers they actually allow. She imagines someone talking as using the EFF but in practice using MA. This shows the deep interpretative issues facing scholars of science concerning how to associate an actual research approach with a research question on the LRQ.

\section{In Favor of (2) Some methodological adaptationists do not use $M A$.}

When we investigate the evolutionary origins of a given trait, as adaptationists we might often prioritize the functional factors, natural and sexual selection, as the factors of most interest in evolutionary research, and we might start with the question, "Does this trait have a function?" If the trait, after investigation, doesn't appear to have a function, correlation with fitness, or design features, we might pursue other evolutionary explanations (Lloyd 2016, 132)

This comes in the context of explaining the EFF approach. Here she says that the EFF can prioritize adaptation and so certain factors over others. It is not clear how prioritizing is different from starting with adaptationism and how these selection-first EFF users avoid the pitfalls of methodological adaptationism. In terms of identification as EFF, it is clear when a program starts with selection and actually ends up supporting another kind of hypothesis. But not all cases are so clear and there will be cases which are not obviously EFF or MA.

Someone following the evolutionary factors framework, asks, quite generally:"What evolutionary factors account for the form and distribution of this trait," or, for example, they can start with "Does this trait have a function?" This question has a series of possible distinct answers (that might be considered in any order, except that the adaptive answers usually go first in practice) (Lloyd 2016, 132)

Here she claims that the EFF allows considering factors in different orders and systematically prioritizing adaptation, which is just how defenders of methodological adaptationism characterize their approach.

We can easily use a more encompassing evolutionary and Darwinian method, the evolutionary factors approach, under which we can ask a variety of questions, including the adaptationist question (Lloyd 2016, 137)

Here she claims that the EFF allows the possibility of asking the adaptationist question. This is the first time she explicitly talks in terms of multiple questions.

So, it is not just that the methodological adaptationists are asking the wrong questions, but in addition, that they are mishandling a possible answer to the question that they are asking, the one that says "this trait does not have a function." At that point, the methodological adaptationist could move on to a different, more productive question, such as "What other evolutionary factors may play a role in evolving this trait?" But, and this is the point, they do not. And this leads to a variety of scientific errors (Lloyd 2016, 142) 
Lloyd again talks in terms of multiple questions and question switching. This is hard to interpret. Of course, MA in the problematic sense means never considering other factors. But what if the program she is imaging did exist, how would we characterize it? There are two possibilities. First, the program which first asked the MA question and the EFF question switched approaches from MA to EFF. Second, the program consistently follows the MA (or EFF) approach, which allows multiple questions and starting with certain factors.

\section{Taking Stock}

Both (1) and (2) have textual support and opposition. Lloyd's official position is (1), that the methodological adaptationists which she has analyzed and critiqued use MA and that this is why their approach should be abandoned in general. In order to challenge horn (1), in the next section I provide a novel case study of successful approaches analogous to adaptationism which in practice move beyond their starting point. My case study further challenges horn (2) by showing that opposing approaches would be both classified as EFF. The LRQ has the consequence that all non-objectionable approaches are EFF. I therefore use my case study to reject the dilemma and suggest a way of enriching the LRQ with more structure to enable finer grade distinctions in approaches.

\section{Two Approaches in Community Ecology}

The LRQ is applicable across the biological sciences and beyond. As Lloyd (2015) says,

While I apply this logic to the adaptationist methodology, my fundamental claim is not about adaptationism exclusively, but rather about how scientific investigation is done, in general, and the "logic of research questions" is thus applicable to any scientific field that experiences controversy about methods and inference. (346)

Community ecology is a natural place to look to evaluate ideas about research programs and methodological approaches because it has so many of them. The diversity and complexity of the systems studied, its disjoint history, and the lack of any general theoretical framework all contribute to methodological diversity in ecology. I examine two approaches analogous to adaptationism: competitionism and neutralism. Extending Lloyd's framework to ecology is straightforward: the Ecological Factors Framework asks, What ecological factors account for the form and distribution of organisms in this community?

\section{Community Ecology: Patterns, Processes, and Heuristics}

Community ecologists are interested in describing, predicting, explaining, and controlling patterns of abundance and diversity of organisms that live in the same place. How much biodiversity is there, and what ecological factors maintain and modify it? Ecological communities occupy the range in between single populations and whole ecosystems. Community ecologists often restrict their domain to single trophic level communities, in order to exclude interactions involving predation and to ensure all species compete for the same kinds of resources (Mittelbach and McGill 2019).

Many of the basic patterns community ecologists study come from counting and sampling (Magurran 2004). Species richness counts record the number of species in a community. Relative species abundance distributions record the number of species and the number of individuals in a community. Ecologists study these patterns both individually (for a single community at 
a single time) and comparatively (across time, space, and scales-from square meter plots to entire continents). Many features of these patterns call for explanation: the number of species increases with habitable area, most species are rare, relative species abundance distributions have a characteristic shape, species correlate with certain resources, not every species lives everywhere it can, etc. Other significant patterns include resource-species correlations, from why certain species colonize open spaces to why conifers live in the taiga.

Ecologists have discovered many causal factors relevant to these patterns at many scales. Mark Vellend (2010, 2016) proposed a theory of ecological communities which unifies the many factors and processes investigated by community ecologists into four high-level processes: drift, dispersal, speciation, and selection. These processes are different ways of changing the composition and abundances of species. Drift is stochastic death and birth; dispersal is how organisms move across space; speciation is how populations of new species arise; and selection is the differential reproduction of certain individuals due to their species. Ecological selection is more commonly known as interspecific competition because it arises from competition between differentially adapted individuals of different species. I will refer to it as just competition.

Different research programs focus on different subsets of Vellend's four processes. Programs interested in descriptive and predictive accuracy can formalize their chosen processes into mathematical models. They can then compare the models with abundance data and evaluate the model fit with statistics. 1 Whether a model fit is good or poor depends on the predictions of the model, the data, and the standards set.

We need to look at three heuristics scientists use to respond to model fits. I describe the heuristics abstractly here and in the next section show how they are used. A few background details about the heuristics first. These are heuristics in the sense of Wimsatt (2007), problem solving strategies with limited scope but great power when used in conditions they are adapted to. These three heuristics address a relative significance problem (Beatty 1997; Kovaka 2017, ch. 5). Relative significance problems concern the relative significance of multiple causal factors or processes to a pattern. The causal relevance of the causal factors or processes to the kind of pattern is accepted. A relative significance problem asks what the contribution of each of the factors is in particular cases. While any relative ratio of significances may be found, the simple way of describing these heuristics use the idea that some subset of processes "dominates" or accounts for more of the data than the remaining processes. Each of the following three heuristics are ways that ecologists have for making inferences about the relative significance.

Heuristic 1: Basic Explanation

From good model fit, conclude that the processes included in the model dominate the data.

This is the basic modeling inference, adapted to the case of relative significance of processes and models containing subsets of processes.

Heuristic 2: Baseline Modeling ${ }^{3}$

From poor model fit, conclude that the processes excluded from the model dominate the data.

The model playing the role of the baseline model is used to detect deviations from the data. These deviations are then ascribed to known or unknown processes excluded from the model. The lack of deviations from the baseline model are ascribed to the processes included in the model. ${ }^{\text {. }}$

\footnotetext{
${ }^{2}$ I trace the straightforward "model fit" to Lloyd (2009).

${ }^{3}$ Baseline modeling is characterized in detail in (Bausman 2018).

${ }^{4}$ I might have said that Baseline Modeling includes Basic Explanation when the model fit is good, but it is useful to keep them distinct as the former is less appreciated.
} 
Heuristic 3: Adding Complexity
From poor model fit, decide to augment the model with (some of) the excluded processes.

The shortcomings of the model are here rectified, not by citing additional processes, but my augmenting the model to include the excluded processes. Adding Complexity describes how models are developed over time, not used for explanations. The augmented models can then be compared with data and followed by any of these heuristics again.

Used in concert, these three heuristics enable research programs to respond productively to any confrontation of models to data and to develop over time.

\section{Competitionism}

Competitionism is the approach based on starting to investigate the role of interspecific competition in patterns of abundance and diversity. Competitionists then use Basic Explanation, Baseline Modeling, and Adding Complexity heuristics to move beyond interspecific competition.

Competitionist research programs tell the following basic story: Different species have different abilities to utilize resources. The species best able to utilize a resource will exclude its competitors from using the resource. In this way, species differences and resource distributions affect species abundances. Competitionism's basic explanatory principle is the competitive exclusion principle: sufficiently similar species cannot coexist because one will out-compete and exclude the other. Their basic investigative heuristic is to seek relevant species differences when coexistence is observed. In this way, the competitionist's starting point is interspecific competition based on species differences.

One competitionist research program is led by David Tilman $(1980,1982)$ and built around his Resource-Ratio, known as $R^{*}$ ("R Star") Theory. The only factor considered in $R^{*}$ Theory is competition between species for limited resources. In $R^{*}$ Theory, each species has a minimum level of a resource (the $R^{*}$ value) that they require to at least maintain their population size. The Theory predicts that, when two species compete for a single limiting resource, the species with the lower $R^{*}$ value will exclude the other species. Species coexistence is possible, for example, when two species compete for two limiting resources and neither species has lower $R^{*}$ s for both resources (see (Miller et al. 2005, Wilson, Spijkerman, and Huisman 2007) for a general review of $R^{*}$ Theory).

An example of a basic explanatory success for this competitionist program is an experiment Tilman (1977) performed for his PhD. Tilman gathered two species of algae from Lake Michigan and measured their respective $R^{*}$ values for both phosphate and silicate inside of a chemostat (a laboratory environment in which the resource level can be kept constant over time). With these four $R^{*}$ values, Tilman made predictions from $R^{*}$ Theory about whether the two algae species would coexist or not in the chemostat given certain levels of phosphate and silicate. Tilman was able to produce both stable coexistence and competitive exclusion in the chemostat by varying the relative ratios of phosphate and silicate, confirming $R^{*}$ Theory. Using the Basic Explanation heuristic, Tilman argues that this the successful predictions of $R^{*}$ Theory show that interspecific competition is responsible for both producing coexistence and competitive exclusion (see also, e.g., Tilman 1986; Stanley Harpole and Tilman 2006).

Yet competitionists are not blind to everything but interspecific competition. Tilman has argued that some cases where his $R^{*}$ Theory fails to give accurate predictions show that additional processes such as immigration are important. For example, Tilman leads the University

๑ OPEN ACCESS - PTPBIO.ORG 
of Minnesota's Cedar Creek Ecosystem Science Reserve, part of the Long-Term Ecological Research network, where he conducts grassland field experiments. Field experiments must control for invasion by wild species. Tilman (1994) argues that some cases where $R^{*}$ Theory poorly predicts abundance patterns actually show the effect of immigration on the experimental plots.

This is an example of the explanatory heuristic Baseline Modeling. The competitionists begin with a theory that includes only interspecific competition and test it. When the model fit is poor, rather than being disconfirmation of $R^{*}$ Theory, the competitionists conclude that other processes such as immigration not included in the model generating the predictions are playing an important role in structuring the observed patterns.

Tilman has developed $R^{*}$ Theory in a new direction due in part to the Invasion Paradox: $\mathrm{Na}$ tive species resist invasion from exotic species. But exotic species also invade and exclude native species. $R^{*}$ Theory explains the former well, but the latter shows the importance of immigration. Tilman's (1994) Stochastic Niche Theory incorporates drift and immigration with resource competition. This is an example of the developmental heuristic Adding Complexity. Starting from only interspecific competition, the competitionists are led to include other processes such as drift in their theoretical toolbox.

\section{Neutralism}

Neutralism is the approach based on starting to investigate the role of drift, immigration, and speciation on patterns of abundance and diversity. Neutralists then use Basic Explanation, Baseline Modeling, and Adding Complexity heuristics to move beyond drift, immigration, and speciation.

Neutralist research programs tell the following basic story: Drift decreases the diversity of a community by slowly driving species extinct. Immigration increases diversity as individuals from the metacommunity, typically with greater diversity, slowly disperse into the local community. In the metacommunity, diversity is maintained against drift via speciation. Moreover, all individuals, regardless of species, are functionally equivalent or neutral. This has the effect of both excluding interspecific competition and making current abundances dependent on past abundances. Abundance patterns result from the relationship of the rates of the three processes of drift, immigration, and speciation in much the same way as the water level of a sink results from the relationship of the inflow and outflow rates. Neutralism's basic explanatory principle is that even functionally equivalent species can coexist indefinitely. Their basic investigative heuristic is to seek the effects of community size, immigration, and speciation when coexistence is observed. In this way, the neutralist's starting point is drift, immigration, and speciation based on species similarities. 5

The best example of a neutralist research program is led by Stephen Hubbell (2001), who developed The Unified Neutral Theory of Biodiversity and Biogeography ("Neutral Theory" for short). ${ }^{6}$ Neutral Theory includes only drift, immigration, and speciation. Neutral mod-

\footnotetext{
${ }^{5}$ Neutrality is logically independent from drift, immigration, and speciation, but they are historically linked. Neutrality alone is not the neutralists's starting point, first, because it is not a process, and second, because neutralists are far more inclined to relax neutrality before they remove drift, immigration, or speciation from their theory or models. Granting their strong Lakatosianism (which both Lloyd's and this paper implicitly challenge), Ale (2019) and colleagues crucially misidentify the "hard core" of the neutrality program as neutrality and their argument for neutralism being a degenerate program fails because of it. See Bausman (2019) for the extended version of this argument.

${ }^{6}$ The history of neutral theorizing and modeling in ecology is complicated and somewhat controversial. In brief, Caswell (1976) imported neutral models from evolution. Then Hubbell (1979; Hubbell and Foster 1986) adapted and applied them to tropical forests. Later, Hubbell published two more papers before his 2001 book, while at the same time Bell $(2000,2001)$ wrote two papers. For more on the history, see Bausman (2016, ch. 1-2).
} 
els are individual-based models which assume neutrality and approximately fixed community sizes. Neutral Theory predicts that species abundance distributions will fit the statistical distributions produced by the neutral model. An example of a basic explanatory success for the neutralist program is its ability to describe the abundance patterns of a tropical forest. Hubbell co-founded the Forest Dynamics Plot Project at the Smithsonian Tropical Research Institute on Barro Colorado Island in 1982. They have complete census data for the 50-hectare plot for every 5 years. The neutral model describes the abundances and dynamics of the BCI forest plot well. Using the Basic Explanation heuristic, Hubbell and colleagues argue that the neutral model's good model fits to that the patterns of abundance of trees on BCI show that these patterns are mainly the result of immigration balancing drift (Condit, Chisholm, and Hubbell 2012).

Neutralists are not blind to the effects of species differences and interspecific competition either. For example, Hubbell showed that while the species abundances in five forest plots from Central America can be explained using neutral models, there is a stronger correlation of the abundances of the commonest species across the five plots than is expected given a neutral model (Hubbell 2001, 337). Because the neutral model leaves out interspecific competition and poorly predicts the abundances of the commonest species, Hubbell argues that their abundances are dominated by interspecific competition. This is an example of the explanatory heuristic Baseline Modeling.

Other neutralists have directly modified neutral models to relax neutrality and introduce interspecific competition. For example, Rosindell, Harmon, and Etienne (2015) use to the neutral model to predict the change in number of species over macro-evolutionary time. However, the neutral model predicts too many species at any given time than we observe in the fossil record. The ecologists therefore relaxed the neutrality assumption and introduced small selective differences into the neutral model. The small degree of interspecific competition included in the nearly neutral model reduces the expected number of species and gives more accurate predictions. This is an example of the developmental heuristic Adding Complexity.

\section{Approaches Order Factors}

\section{Why Community Ecology is a Problem for the LRQ}

The case of competitionism and neutralism is an example of relevantly different methodological approaches which are both open to considering, and have actually considered, all relevant factors. A philosophical account of methodological approaches should be able to account for this, but the LRQ cannot do so as it currently stands. To see this, let us ask how the LRQ should characterize competitionism and neutralism in comparison with the MA and (ecological) EFF. This leads to a second dilemma:

\section{Dilemma 2}

(3) Competitionism and neutralism are analogs of MA.

\footnotetext{
${ }^{7}$ The neutral model has been claimed to supply the appropriate "null hypothesis" with respect to competition hypotheses. This is exact opposite illegitimate strategy to the one Lloyd $(2015,353)$ characterizes. In Lloyd's case, causal theories are called a null hypothesis in order to disqualify them from providing causal explanations. In the neutral theory's case, a causal theory is called a null hypothesis in order to give it the benefit of the doubt with respect to causal explanations. I agree with Lloyd that neither case is doing statistical null hypothesis testing and both lines of argument are unjustified. See Bausman and Halina (2018) for the opposite case to Lloyd's involving neutral theory and the behavior reading hypothesis in comparative psychology.
} 
(4) Competitionism and neutralism are each versions of the EFF.

On horn (3), competitionism and neutralism are analogs of MA because they each investigate a subset of ecological factors and do not treat all factors equally. These approaches are distinct from EFF and from each other. One consequence of taking horn (3) is that competitionism and neutralism rise and fall with methodological adaptationism. Horn (3) goes together naturally with horn (1) above. All three approaches should be shunned in favor of EFFs. On horn (4), competitionism and neutralism are versions of the EFF because they exclude no factors from their investigation. One consequence of taking horn (4) is that both research programs use the same approach. In fact, all acceptable approaches are the same approach.

Horn (3) is unacceptable, first, because neither competitionists nor neutralists exclude any subset of factors from their full investigation. competitionists sometimes invoke drift, immigration, and speciation; neutralists sometimes invoke interspecific competition. Further, (3) is unacceptable because the competitionists and neutralists run successful research programs that persist against calls for unity within ecology. This suggests they are useful and shifts the burden of proof to find a problem particular to each of them individually. Until such a problem is identified, the approaches that successful programs use should be seen as acceptable.

Horn (4) is unacceptable because it lumps together distinct approaches. Competitionism and neutralism go about their investigation in mutually opposed directions and they should not both be characterized as EFF. Further, a satisfactory account of research questions and approaches should distinguish between approaches which take a starting point and approaches which have no systematic order to their research.

If forced to choose, I suspect the proponent of the LRQ view would choose horn (4) and bite the bullet that competitionists and neutralists use the same approach qua LRQ perhaps viewing them as two versions of the same approach. This puts tension on choosing horn (1) of Dilemma 1 , and I expect they would now choose horn (2) should analogous instances of methodological adaptationist programs in evolutionary biology be produced. But both horns (2) and (4) seem best only given that they are better than their alternatives. The common assumption of both dilemmas is that methodologies must be characterized solely in terms of single questions and the factors considered.

\section{The Ordered Factors Proposal}

Drawing on the importance of starting points, the LRQ needs to consider the way that approaches order their investigation of questions and factors. The Ordered Factors $(+\mathrm{OF})$ proposal extends the LRQ to represent approaches using sequences of research questions or factors, the ordered sequences corresponding to the order of inquiry. The evolutionary and ecological factors frameworks characterizations remain unchanged. Now we can distinguish dogmatic methodological adaptationism from sophisticated methodological adaptationism. Dogmatic adaptationism uses MA. Sophisticated adaptationism can be formulated as:

\section{Sophisticated Adaptationism}

1. How can natural selection account for the form and distribution of this trait?

2. How can other evolutionary causes account for what natural selection cannot?

Accordingly, the approaches in community ecology can be formulated as:

Competitionism

๑ OPEN ACCESS - PTPBIO.ORG 
1. How can interspecific competition account for the distribution and abundance of organisms?

2. How can drift, immigration, and speciation account for what interspecific competition cannot?

\section{Neutralism}

1. How can drift, immigration, and speciation account for the distribution and abundance of organisms?

2. How can interspecific competition account for what interspecific competition cannot?

Because with the LRQ, research questions imply their answers and their answers are just sets of causes or factors which are acceptable answers to the question, to shorten their description we can also represent approaches as sets of causes or factors, as in Table 1. This allows comparison of the various options of the views at a glance. Let round brackets mark ordered sequences and curly brackets mark unordered sets.

\begin{tabular}{ccccc}
\hline & \multicolumn{2}{c}{ Competitionism } & \multicolumn{2}{c}{ Neutralism } \\
\hline Horn & $(3)$ & $(4)$ & $(3)$ & $(4)$ \\
LRQ & $\{\mathrm{C}\}$ & $\{\mathrm{D}, \mathrm{I}, \mathrm{S}, \mathrm{C}\}$ & $\{\mathrm{D}, \mathrm{I}, \mathrm{S}\}$ & $\{\mathrm{D}, \mathrm{I}, \mathrm{S}, \mathrm{C}\}$ \\
$+\mathrm{OF}$ & $(\mathrm{C},\{\mathrm{D}, \mathrm{I}, \mathrm{S}\})$ & $(\{\mathrm{D}, \mathrm{I}, \mathrm{S}\}, \mathrm{C})$ \\
\hline
\end{tabular}

Table 1: Competitionism and Neutralism

In characterizing a methodology with $+\mathrm{OF}$, we need not find a linear ordering of all the factors considered. Neither adaptationism, nor competitionism, nor neutralism linearly orders all the factors it considers. Some methodologies may have no general ordering of their factors. They may have heuristics for when to apply one ordering and when to apply another. The + OF proposal does not impose order on the programs, it captures the ordering present in the methodology.

\section{Why Starting Points}

Methodologies order factors because orderings are useful for biologists addressing relative significance problems. Competitionists and neutralists do argue sometimes as if they are engaged in a theory choice problem between whether interspecific competition or drift, immigration, and speciation is the one true set of processes responsible for abundance patterns. But biologists' debates are not usually like the exclusive choices found in the Geocentrists vs. Heleocentrists. While biologists often argue in terms of why their pet processes are the most important processes, their actions show that they acknowledge that all the known factors are in play. Starting points help biologists to answer these relative significance problems.

By imposing an ordering on their inquiry, when biologists decide that they can no longer make do, they move beyond their starting point. As I showed with the competitionist and neutralist programs, biologists have at least two heuristics for this. In Baseline Modeling the

\footnotetext{
${ }^{8}$ These two features do not exhaust methodologies. One need only find two programs which have distinct approaches, but which share the same factors considered and ordering to see this.
}

๑ OPEN ACCESS - PTPBIO.ORG 
processes excluded from the models and incorporated directly into the explanation of the pattern. The reason neutral models offer bad predictions of, say, which species of paramecium will exclude the other when they are isolated and given a constant level of nutrient is because interspecific competition is responsible for this exclusion. By finding that you cannot do without citing interspecific competition, you have better evidence that interspecific competition is playing an important role than if you merely made good predictions from a competition model. And similarly for Adding Complexity.

Bill Wimsatt (1987) argues that the initial assumptions research programs make do not directly aim at the truth but are strategic decisions about how to get closer to the truth later. This fits how many biologists describe their methodologies. As Tilman (1986) writes:

Thus, many of the broad, long-term patterns observed in plant communities may be the result of interspecific competition for limiting resources. Such correspondence does not mean that other processes are unimportant in structuring these plant communities, but the simple theory that predicts them may provide a framework for more detailed studies. (75)

Hubbell (2008) similarly writes that

the most rapid path to understanding ecological systems ... is through an interaction of empirical science ... with theories that start very simply, with few free parameters and assumptions, and add complexity reluctantly, kicking and screaming, only when absolutely necessary to obtain some desired level of fit to the data. Neutral theory is one such starting point. (143)

Lloyd cites Ernst Mayr (1983) writing

[The evolutionary biologist] must first attempt to explain biological phenomena and processes as the product of natural selection. Only after all attempts to do so have failed, is he justified in designating the unexplained residue tentatively as a product of chance.

She is right to press Mayr for waiting too long to look for alternative explanations, but in doing so she throws out the characteristic feature of adaptationism: taking natural selection as its starting point.

Given that starting points are useful, it is a further interesting question why there is an extant plurality of them across biological disciplines. Fully answering this question goes beyond the scope of this paper. But here is a clue. In the examples from ecology, the most important factor shaping each methodology is the initial target system of the program. 0 . Competition theory was built around creating artificial situations in a laboratory where a small number of species interact for controlled resources. 10 Neutral theory was built around counting trees in tropical forests where ecologists have no control. Arguably, each program grew from systems in which their starting point processes dominate the other known processes. Over their development, these programs were driven beyond their starting points by the failure of nature to abide with what worked earlier.

\footnotetext{
${ }^{9}$ Other significant factors shaping methodology include: the question asked, the traditions one works in, observational and experimental techniques and instruments, disciplinary constraints, personalities, etc.

${ }^{10}$ This does not fit the biogeographical work of Grinnell, Elton, MacArthur, and Diamond on birds.
} 


\section{Objections and Responses}

\section{Lack of Stopping Rules}

In characterizing the LRQ Lloyd anticipates the proposal I defend. Her strongest point against the $+\mathrm{OF}$ proposal is her denial of the existence of stopping rules. She argues adaptationists like Mayr, who say they only start with natural selection before moving to additional factors, cannot actually move on because they lack rules for when to stop looking for a given factor. This is not the statistician's concept of a rule for when to stop collecting data and begin analyzing it. The concern is that, without formal standards for when to abandon the search for the function of a trait, adaptationists will under no circumstances consider alternative explanations. This would entail that starting points are not useful because they would not enable heuristics such as Baseline Modeling and Adding Complexity.

My response to this is twofold. First, this is not a special problem for adaptationists. Any methodology which entertains working hypotheses they are willing to revise faces the problem of whether to tinker with or abandon a given hypothesis that has not met the standards of evidence. The evolutionary factors framework also lacks a stopping rule for make decisions such as: Did this developmental constraint hypothesis perform worse than a sexual selection hypothesis because development was truly the better explanation or because they had not stated the developmental constraint hypothesis good enough?

Second, I deny that formal, algorithmic stopping rules are required. Scientists have only heuristics for when to move on. Good judgment should decide when enough failure is enough, and the development of a research program involves risk and trial and error. I agree with Lakatos that judgements about the health of a research program are only justified in hindsight after a long run.

\section{Adaptationism Is Not Useful}

Lloyd (2015) almost explicitly addresses the use of starting points in one place:

I do not mean to deny the common point about the division of scientific labor, by saying that it is a good idea for some to start by asking about the function of a trait .... The question is: is it useful for anyone to be a methodological adaptationist rather than following an evolutionary factors approach? (348, fn. 32)

She answers 'no' because she does not allow that methodological adaptationists can systematically entertain the factors included in the evolutionary factors view while imposing an ordering on the factors. But without recourse to the lack of stopping rules, this cannot be maintained. I have shown how starting points are useful, in part because they enable powerful heuristics for moving beyond one's starting points. Further, the continued existence of adaptationism, competitionism, neutralism, and the many other research programs organized around a subset of factors in the hostile environments that are their larger disciplines is a good sign that such methodologies are useful in general. Specific criticisms are needed to undermine specific programs.

\section{Division of Labor}

In the last quotation, Lloyd also suggests that starting points are not enough to distinguish approaches because they are variations within one methodology engaged in "the division of scientific labor". This response portrays research programs like the competitionists and neutralists 
as engaged as parts of a larger, unified program using the Ecological Factors Framework. But these programs do not only mutually benefit each other while working toward common goals. They work on only partially overlapping questions and offer competing answers to them. Programs in ecology interact in ways analogous to how species interact in a community.

\section{Methodological Pluralism}

Lurking beneath the difference between the LRQ and $+\mathrm{OF}$ is a deep philosophical question about scientific methodology. The question is, How many acceptable methodological approaches are there for a given domain of biology? Methodological monists answer "one", methodological pluralists answer "many". It is unclear from her writing whether Lloyd is a methodological monist in evolution. But one reason for suspecting she might be is that, as I have argued in this paper so far, the Evolutionary Factors Framework seems to be the only acceptable evolutionary approach. There are multiple approaches, but there can be only one good approach with the LRQ because only the EFF approach encompasses all the evolutionary factors.

Lloyd positions herself as a pluralist against the monistic and dogmatic adaptationists. But in her lumping together of what I have argued are acceptable forms of methodological adaptationism under the EFF, I see a monistic inclination. My + OF proposal in contrast supports methodological pluralism and diversity. This section draws out this monistic consequence of her treatment of MA and EFF in evolution by relating it to her previous work on the levels of selection in order to better understand her views.

By recognizing that approaches order factors, we can distinguish methodological monism/ pluralism from factor monism/pluralism. Lloyd is a factor pluralist because she argues, against the factor monism of dogmatic adaptationists, that any good approach must be open to considering all relevant factors. But again, she seemingly argues further that there can be only one approach in evolution - the EFF — and so for methodological monism.

Lloyd's position on factors and approaches mirrors her position on a question about the levels of selection, whether natural selection operates at the level of genes (Genic Selection View) or at the levels of genes and organisms (Multi-level Selection View). Lloyd argued that once the multi-level selection view is accepted, it would follow that genic models would stop being used (Lloyd 2005). She argued that all processes known to be a causally relevant to a pattern should be incorporated into all models used to fit that pattern. Ken Waters responded that there are tradeoffs associated with using genic and multi-level selection models and that each model is appropriate some of the time (Waters 2005). Therefore, we should not expect genic models to go away. Waters characterized his position as "pluralism about levels of selection" and Lloyd's as "monism about the levels of selection", but the distinction concerns the usefulness of one vs. many kinds of model of selection, because both sides acknowledge selection can operate at multiple levels. 11 I will refer to these positions as model monism and model pluralism, respectively.

The Genic Selection View supports level monism while the Multi-level Selection View supports level pluralism. Lloyd believes that natural selection operates at multiple levels (level pluralism) and further argues that the only good models of natural selection are multi-level selection models (model monism). This is analogous to how that she believes that all the many evolutionary factors beyond natural selection need to be included in any evolutionary approach (factor pluralism) and further argues that the only good evolutionary approach is the EFF (methodological monism). Just as all levels of selection should be incorporated into all models of selection,

\footnotetext{
${ }^{11}$ It is worth noting that Lloyd responded that she is also a pluralist and that "Waters seems to think that he and his coauthors hold a monopoly on the term 'pluralism." (Lloyd et al. 2005) Nevertheless, I agree with Waters that model and methodological pluralisms are the more interesting and challenging forms of pluralism.
}

๑ OPEN ACCESS - PTPBIO.ORG 
so too should all factors be included into the one and only approach. I accept factor pluralism about biological factors and further advocate methodological pluralism for the same reason Waters advocates model pluralism, namely that multiple approaches, each taking a subset of factors as their starting point, are good strategies to investigate biological systems.

This discussion of pluralism concerns Lloyd's application of the LRQ to evolution. She has also applied the LRQ to a methodological dispute in climate science (Lloyd and Oreskes 2018). Here she and Naomi Oreskes defend the emergence of the new storyline approach of and its continued existence alongside the preexisting risk-based approach. Without going into detail, I view this as a successful application of the LRQ to characterize and distinguish methodological approaches. And it shows that Lloyd should not be characterized as a methodological monist in every domain.

But this successful application does not mean that the arguments I have made in this paper do not stand. First, my +OF proposal is not committed to the consequence that no two acceptable methodological approaches in a given domain can be distinguished by questions and factors alone. The climate science case seems to be a case where they can be. Second, the climate science alternative methodologies are therefore differently different than the methodological approaches I have considered here in evolution and ecology. In my view, the relevant difference is that the approaches Lloyd and I discuss in evolution and ecology require distinguishing the order in which factors are considered. I presume that other approaches in evolution and ecology can be characterized, as in climate science, using only the LRQ. But I have argued in this paper that methodological adaptationism and its ecological analogs neutralism and competitionism cannot be. And third, this climate methodology application corroborates my view that the LRQ is capable of and that Lloyd uses to neutrally characterize methodologies in terms of questions and factors, not only to criticize problematic applications.

\section{Conclusion}

Adaptationism and analogous methodologies consider their preferred factor first. While some dogmatic programs crudely fail to look beyond their starting point, this is not an essential feature of all adaptationist approaches. We therefore need to enrich the Logic of Research Questions framework needs to characterize methodologies at the scale of adaptationism, competitionism, and neutralism. These programs take a methodological starting point that enables heuristics like Baseline Modeling and Adding Complexity to give direction to their research. We should understand approaches not only in terms of the factors they are open to considering but also the order in which they will consider them. The Ordered Factors Proposal shows that characterizing research questions remains a useful tool for understanding local research activities of research programs in particular contexts. Research programs ask many questions depending on their goal, their background knowledge, and their starting point.

\section{Acknowledgments}

I presented earlier versions of this paper to the Lake Geneva Biological Interest Group and at POBAM 2018 in Salt Lake City and I thank the participants there for their comments and questions. I also thank Max Dresow, Elisabeth Lloyd, Shay Logan, María José Ferreira Ruiz, Ken Waters, and Marcel Weber for comments on earlier versions of this manuscript. I was supported in writing this article in part by grant 50191 from the John Templeton Foundation, From Biological Practice to Scientific Metaphysics. 


\section{Literature cited}

Ale, Som B., Abdel Halloway, William A. Mitchell, and Christopher J. Whelan. 2019. "Does God Roll Dice? Neutrality and Determinism in Evolutionary Ecology." Biology \& Philosophy 34 (1): 3.

Bausman, William C. 2016. "Neutral Theory, Biased World.” PhD diss., University of Minnesota.

Bausman, William C. 2018. "Modeling: Neutral, Null, and Baseline." Philosophy of Science 85 (4): 594-616 doi: 10.1086/699021.

Bausman, William C. 2019. "The Aims and Structures of Ecological Research Programs.” Philosophical Topics 47 (1).

Bausman, William, and Marta Halina. 2018. "Not Null Enough: Pseudo-Null Hypotheses in Community Ecology and Comparative Psychology." Biology \& Philosophy 33 (3): 30. doi: 10.1007/s10539-018-9640-4.

Beatty, John. 1997. “Why Do Biologists Argue like They Do?” Philosophy of Science 64 (Proceedings):S432-S443.

Bell, Graham. 2000. "The Distribution of Abundance in Neutral Communities." The American Naturalist 155 (5): 606-617.

Bell, Graham. 2001. "Neutral Macroecology.” Science 293 (5539): 2413-2418.

Caswell, Hal. 1976. "Community Structure: A Neutral Model Analysis." Ecological Monographs 46 (3): 327-354.

Condit, Richard, Ryan A. Chisholm, and Stephen P. Hubbell. 2012. "Thirty Years of Forest Census at Barro Colorado and the Importance of Immigration in Maintaining Diversity." PloS One 7 (11): e49826.

Gould, Stephen Jay, and Richard C. Lewontin. 1979. "The Spandrels of San Marco and the Panglossian Paradigm: A Critique of the Adaptationist Programme." Proceedings of the Royal Society of London B: Biological Sciences 205 (1161): 581-598.

Hubbell, Stephen P. 1979. "Tree Dispersion, Abundance, and Diversity in a Tropical Dry Forest." Science 203 (4387): 1299-1309.

Hubbell, Stephen P. 2001. The Unified Neutral Theory of Biodiversity and Biogeography. Monographs in Population Biology, edited by Simon A. Levin and Henry S. Horn. Princeton: Princeton University Press.

Hubbell, Stephen P. 2008. "Approaching Ecological Complexity from the Perspective of Symmetric Neutral Theory." In Tropical Forest Community Ecology, edited by Walter Carson and Stefan Schnitzer, 143-159. Chichester, UK: Wiley-Blackwell.

Hubbell, Stephen P., and Robin B. Foster. 1986. "Biology, Chance, and History and the Structure of Tropical Rain Forest Tree Communities." In Community Ecology, edited by Jared Diamond and Ted J. Case, 330. Harper \& Row.

Kovaka, Karen. 2017. "Understanding Innovation and Imitation in Evolution.” PhD diss., University of Pennsylvania.

Lloyd, Elisabeth A. 2005. "Why the Gene Will Not Return.” Philosophy of Science 72 (2): 287-310.

Lloyd, Elisabeth A. 2009. "Varieties of Support and Confirmation of Climate Models." Aristotelian Society Supplementary Volume 83 (1): 213-232.

Lloyd, Elisabeth A. 2015. "Adaptationism and the Logic of Research Questions: How to Think Clearly About Evolutionary Causes.” Biological Theory 10: 343-362. 
Lloyd, Elisabeth A. 2016. "What a Difference Research Questions Can Make!" American Philosophical Association, Pacific Division.

Lloyd, Elisabeth A., Matthew Dunn, Jennifer Cianciollo, and Costas Mannouris. 2005. "Pluralism Without Genic Causes?" Philosophy of Science 72 (2): 334-341.

Lloyd, Elisabeth A., and Naomi Oreskes. 2018. "Climate Change Attribution: When Is It Appropriate to Accept New Methods?” Earth's Future 6 (3): 311-325.

Magurran, Anne E. 2004. Measuring Biological Diversity. Blackwell Science.

Mayr, Ernst. 1983. "How to Carry Out the Adaptationist Program?” American Naturalist: 324-334.

Miller, Thomas E., Jean H. Burns, Pablo Munguia, Eric L. Walters, Jamie M. Kneitel, Paul M. Richards, Nicolas Mouquet, and Hannah L. Buckley. 2005. "A Critical Review of Twenty Years' Use of the Resource-Ratio Theory." The American Naturalist 165 (4): 439-448.

Mittelbach, Gary G., and Brian J. McGill. 2019. Community Ecology: Oxford University Press.

Rosindell, James, Luke J. Harmon, and Rampal S. Etienne. 2015. “Unifying Ecology and Macroevolution with Individual-Based Theory.” Ecology Letters 18 (5): 472-482.

Stanley Harpole, W., and David Tilman. 2006. "Non冈neutral Patterns of Species Abundance in Grassland Communities.” Ecology Letters 9 (1): 15-23.

Tilman, David. 1977. "Resource Competition between Plankton Algae: An Experimental and Theoretical Approach.” Ecology 58 (2): 338-348.

Tilman, David. 1980. "Resources: A Graphical-Mechanistic Approach to Competition and Predation." American Naturalist: 362-393.

Tilman, David. 1982. Resource Competition and Community Structure. Monographs in Population Biology, edited by Simon A. Levin and Henry S. Horn. Princeton: Princeton University Press.

Tilman, David. 1986. "Resources, Competition and the Dynamics of Plant Communities." In Plant Ecology, edited by M. Crawley, 51-75. Oxford, England: Blackwell Scientific Publications.

Tilman, David. 1994. "Competition and Biodiversity in Spatially Structured Habitats." Ecology 75 (1): 2-16.

Tilman, David. 2004. "Niche Tradeoffs, Neutrality, and Community Structure: A Stochastic Theory of Resource Competition, Invasion, and Community Assembly." Proceedings of the National Academy of Sciences of the United States of America 101 (30): 10854-10861.

Vellend, Mark. 2010. "Conceptual Synthesis in Community Ecology." The Quarterly Review of Biology 85 (2): 183-206.

Vellend, Mark. 2016. The Theory of Ecological Communities. Monographs in Population Biology, edited by Simon A. Levin and Henry S. Horn. Princeton: Princeton University Press.

Waters, C. Kenneth. 2005. "Why Genic and Multilevel Selection Theories are Here to Stay." Philosophy of Science 72 (2): 311-333.

Wilson, J. Bastow, Elly Spijkerman, and Jef Huisman. 2007. "Is There Really Insufficient Support for Tilman's $R^{*}$ Concept? A Comment on Miller et al.” The American Naturalist 169 (5): 700-706.

Wimsatt, William C. 1987. "False Models as Means to Truer Theories." In Neutral Models in Biology, edited by Matthew H. Nitecki and Antoni Hoffman, 23-55. New York: Oxford University Press.

Wimsatt, William C. 2007. Re-Engineering Philosophy for Limited Beings: Piecewise Approximations to Reality. Harvard University Press.

(C) 2022 Author(s)

This is an open-access article distributed under the terms of the Creative Commons Attribution 4.0 
International license, which permits anyone to download, copy, distribute, display, or adapt the text without asking for permission, provided that the creator(s) are given full credit.

ISSN 2475-3025 